\title{
CONSUMO DE ALCOHOL Y VIOLENCIA EN CONTRA DE LA MUJER EN LA EMERGENCIA SANITARIA DE LA COVID-19
}

\author{
ALCOHOL CONSUMPTION AND VIOLENCE AGAINST \\ WOMEN DURING THE COVID-19 HEALTH EMERGENCY
}

Victoria Elizabeth Bueno Ayala ${ }^{1}$

Recibido: 2021-02-26 / Revisado: 2021-04-24 / Aceptado: 2021-05-10 / Publicado: 2021-07-01

Forma sugerida de citar: Bueno-Ayala, V. E. (2021). Consumo de alcohol y violencia en contra de la mujer en la emergencia sanitaria de la COVID-19. Retos de la Ciencia. 5(11), pp. 25-35. https://doi.org/10.53877/rc.5.11.20210701.03

\section{RESUMEN}

El estudio del consumo de alcohol y la violencia contra la mujer en la emergencia sanitaria de la COVID - 19 puso en evidencia la violación de los derechos de la mujer, aún más, dentro del contexto de pandemia. El alcohol es considerado como una bebida social y es una droga aceptada como legal, es más, no es reconocida por el común de la población como droga, por lo que, se ha normalizado su consumo. Sin embargo, estudios realizados a nivel mundial han demostrado un alto índice de mortalidad y de conductas violentas asociadas a la ingesta de la sustancia, los resultados evidenciaron mayor frecuencia de agresión de hombres hacia mujeres. Países a nivel mundial fueron afectados por el virus COVID-19, con la finalidad de preservar la seguridad de los habitantes, los gobernantes decidieron el confinamiento obligatorio para la población, el permanecer en el hogar incrementó los factores de riesgo en los cuales la seguridad de la mujer se vio afectada; ante esta realidad, instituciones gubernamentales ofrecieron diferentes alternativas de protección, a pesar de eso la cantidad feminicidios aumentaron.

Palabras clave: alcohol, mortalidad, violencia, feminicidios.

\section{ABSTRACT}

The study of alcohol consumption and violence against women in the health emergency of COVID 19 revealed a violation of women's rights, even more so, within the context of a pandemic. Alcohol is considered as a social substance and it is a drug accepted as legal, furthermore, it is not recognized by the average person as a drug,

1Psicóloga Clínica. Estudiante de la Maestría en Intervención Psicológica en Adicciones de la Facultad de Ciencias Psicológicas de la Universidad Central del Ecuador Ecuador. Ecuaor. Psicóloga Clínica Ministerio de Salud Publica. Ecuador. Email: vebueno@uce.edu.ec / ORCID: https://orcid.org/0000-0002-4907-4866 
therefore, its consumption has been accepted as a normal behavior. However, studies carried out worldwide have shown a high rate of mortality and violent behaviors associated with the intake of the substance, the results showed a higher frequency of aggression by men towards women. Countries worldwide were affected by the COVID19 virus, in order to preserve the safety of the inhabitants, public elected officials decided the mandatory confinement of the population, staying at home increased the risk factors in which the safety of woman was affected. Faced with this reality, government institutions offered different safety alternatives, despite of the increased number of femicides.

Keywords: alcohol, mortality, violence, femicide.

\section{INTRODUCCIÓN}

Actualmente el mundo vive una nueva realidad, generada por la situación epidemiológica, a causa de la COVID-19, cuyas siglas hacen referencia a la enfermedad del Coronavirus que se desata en diciembre del 2019 como epidemia y hasta marzo del 2020 con carácter de pandemia, esto movilizó la decisión de los gobernantes a decretar confinamiento para proteger la salud de sus habitantes. Sin embargo, este encierro obligado, visibiliza con fuerza la violencia ejercida contra la mujer presentando datos importantes a nivel mundial y regional; al respecto del confinamiento Lorente (2019), afirma que el encierro crea el ambiente necesario para que se desarrolle cualquier tipo de violencia ya que al estar la victima aislada de su círculo social (familia, amigos, vecinos), el agresor utilizará su poder.

La pandemia ha agudizado los problemas asociados a factores políticos, económicos, laborales y sociales, alterando en mayor proporción el bienestar emocional de la población, y como consecuencia el incremento del consumo de bebidas alcohólicas, lo que puede desatar más actos violentos, ante esto la Organización Panamericana de la Salud OPS (2020), afirma que "el riesgo de la violencia sube cuando los hombres beben copiosamente" (párr. 6).

El presente trabajo, tiene como propósito informar a la población lectora sobre la realidad del consumo de alcohol y la violencia contra la mujer, que ha incrementado durante la pandemia. La interrogante que se plantea es ¿Cuáles son los problemas específicos encontrados en el consumo de alcohol y la violencia contra la mujer durante la emergencia sanitaria de la COVID-19?, el objetivo general es analizar investigaciones llevadas a cabo sobre el consumo de alcohol y la violencia contra la mujer durante la emergencia sanitaria COVID-19; esto debido a la importancia que tiene en la salud y en la seguridad integral de la población.

El presente trabajo es una revisión bibliográfica, que tiene coherencia con la investigación cualitativa, como señala Castillo-Bustos (2021), "Teniendo en cuenta que la investigación cualitativa se desarrolla a partir de la observación directa a las situaciones o fenómenos de estudio o desde la experiencia vivida del investigador en el lugar de los hechos" (p. 54). Para tal efecto, se han utilizado como referencia estudios anteriores a la pandemia respecto al consumo de alcohol y violencia contra la mujer, este estudio previo sirve de precedente para abordar la realidad del consumo y la violencia contra la mujer durante la COVID-19. La información recopilada esta argumentada en la búsqueda de artículos publicados en revistas, artículos científicos, publicaciones de la Organización Mundial de la Salud (OMS) y la Organización Panamericana de Salud (OPS), así como experiencias personales profesionales; al 
ser un tema actual, existe poca bibliografía, de tal manera que para poder presentar información sobre Ecuador se acudió a la fuente El comercio.

\section{DESARROLLO}

\section{Consumo de alcohol}

El consumo de alcohol es la ingesta de la sustancia que causa cambios en el organismo a nivel biológico, psicológico, social, y puede poner en peligro la vida del individuo como de terceras personas, sin distinción de la cantidad ingerida, La clasificación estadística internacional de enfermedades y problemas relacionados con la salud CIE10 (1990) define al uso nocivo de alcohol, como un patrón de consumo de la sustancia que causa daño a nivel físico y mental (p.305). Comúnmente se observa a personas con problemas conductuales, alteración en los estados emocionales, cambios en las funciones cognitivas superiores como la atención, concentración, memoria, juicio, añadido a esto problemas en la coordinación y el habla, de esta manera existen consecuencias perjudiciales para el bebedor y su entorno, al respecto OMS (2018) refiere: "En el 2016, el consumo nocivo de alcohol causó unos 3 millones de muertes (5,3\% de todas las muertes) en el mundo" (p.8).

El alcohol es la droga más utilizada, su consumo es considerado un problema de Salud Pública y responde a factores multicausales, comúnmente denominados factores de riesgo, entre ellos factores biológicos, psicológicos y sociales; por una parte los factores biológicos están relacionados con la predisposición genética, es decir, si los padres son bebedores es muy probable que el hijo lo sea, estos factores están asociados a los problemas de salud que el consumidor puede desarrollar a corto plazo por otro lado, los factores psicológicos son elementos individuales, ligados a cogniciones y emociones y los factores sociales tienen que ver con la influencia que ha recibido el individuo del contexto, si la persona vive en un ambiente de riesgo aumenta la probabilidad del consumo. El consumo de alcohol es perjudicial para la salud, los factores de riesgo se encuentran presentes desde la primera ingesta y las consecuencias no discriminan el uso que se le dé, el riesgo de muerte por la ingesta dependiente es elevado, así como la afección a su entorno social. La OMS (2018) informa que:

"De todas las muertes atribuibles al alcohol, el $28 \%$ se debieron a lesiones, como las causadas por accidentes de tránsito, autolesiones y violencia interpersonal; el $21 \%$ a trastornos digestivos; el 19\% a enfermedades cardiovasculares, y el resto a enfermedades infecciosas, cánceres, trastornos mentales y otras afecciones" (párr. 4).

Las bebidas alcohólicas son de venta libre, con diferentes costos y por ende de fácil acceso. El consumo inicia en la adolescencia, el interés por socializar, pertenecer a un grupo y ser aceptados en su medio son factores que inciden en esta problemática. Según estudios realizados por la Organización Mundial de la Salud, el género masculino es la población que más consume:

OMS (2018):

La prevalencia de los episodios de consumo excesivo de alcohol es particularmente elevada en los hombres. En todas las regiones de la OMS, hay menos mujeres que sean consumidoras actuales que hombres; y cuando lo son, beben menos que los hombres (p.12).

A nivel mundial se ha trabajado en la prevención del consumo, sin resultados positivos; algunas de las estrategias para reducir el consumo y la edad de inicio al 
consumo ha sido la prohibición de la venta a menores de edad, infracciones monetarias al conducir en estado etílico, la disminución en el tiempo de apertura de lugares de diversión como bares y discotecas, medidas que no se acatan, el colectivo ha implantado la idea de que no se puede divertir sin consumo de alcohol, mientras tanto grandes empresas siguen auspiciando a deportistas y eso es observado a través de los medios de comunicación a la población general, la OMS realiza una proyección de consumo de alcohol por habitante en el mundo para el año 2025 en las regiones de América, Asia, Sudoriental y del Pacífico Occidental. OMS (2018); "El consumo total de alcohol por habitante en el mundo puede ascender a 6,6 litros en los 2020 y 7,0 litros en el 2025" (p.7). Como se puede observar, se estima que el porcentaje aumente, por lo tanto, las consecuencias perjudiciales para el entorno.

\section{La violencia contra la mujer}

Históricamente la mujer ha sido subordinada, tal es así que la mujer antiguamente era educada específicamente para la atención del esposo y su familia; al hombre por otro lado se le ha atribuido el poder, la fuerza, el concepto de superioridad, se ha reconocido su nivel intelectual, por lo tanto, tuvo acceso a la educación, para responder a las necesidades culturales y económicas en su rol social y familiar. A pesar de que el tiempo ha transcurrido y la mujer ya es reconocida en su rol activo dentro de la sociedad, aún el porcentaje de violencia de la que es víctima es elevado; al respecto la ONU (2012) afirma que: "La violencia contra mujeres y niñas es una de las violaciones de los derechos humanos más generalizadas. En algunos países, hasta 7 de cada 10 mujeres sufrirán golpes, violaciones, abusos o mutilaciones a lo largo de sus vidas" (párr.1), miles de mujeres son discriminadas, humilladas, ultrajadas y violentadas. La Organización de las Naciones Unidas ONU, define la violencia contra la mujer como:

Todo acto de violencia basado en la pertenencia al sexo femenino que tenga o pueda tener como resultado un daño o sufrimiento físico, sexual o psicológico para la mujer, así como las amenazas de tales actos, la coacción o la privación arbitraria de la libertad, tanto si se producen en la vida pública como en la vida privada (ONU, 1993, párr. 14).

La violencia sexual es todo acto que vulnera la sexualidad y que se realiza sin el consentimiento de la persona, con utilización de la fuerza física y amenazas, incluido en esto el estado civil de la mujer, en la mayoría de casos el desconocimiento ha hecho que mujeres sean violentadas sexualmente por sus esposos, novios, o acompañantes de una unión libre, ahí se pone en evidencia la manipulación del hombre tras el autoritarismo y el machismo envueltos en situaciones de celos que hacen comúnmente que las mujeres acepten este tipo de violencia como normal y no sea denunciada.

La violencia psicológica según lo definen Bonifaz y Spanó (2014): "Toda acción u omisión que causa daño a la autoestima, a la identidad o al desenvolvimiento de la persona(...) llegando a considerarse culpable de las acusaciones y responsable por la violencia sufrida" (p.436). Esto explica que muchas veces las mujeres violentadas acepten como verdad información que disminuye su autoestima tales como juzgamiento a su cuerpo y manera de pensar, entender que nadie más las va a amar, que son poco inteligentes, que no podrán salir adelante solas con sus hijos, que si denuncian o abandonan el hogar sus hijos serán retirados de su cuidado, entre otras cuestiones que hacen que no se denuncie por miedo a que se cumpla con toda la información que entrega su pareja. 
La Violencia física es todo acto intencional sea con la utilización de la fuerza corporal o cualquier objeto que causa lesiones; mujeres que son violentadas físicamente ya han pasado seguramente por periodos de violencia psicológica y ya han aprendido un discurso adecuado para justificar los golpes visibles en su cuerpo, ante esto el femicidio puede ser considerado como el acto de mayor violencia en contra de la mujer realizada por el hombre, según el Comercio (2019), en el ecuador se registraron más de 60 femicidios de los cuales el $62 \%$ de los casos fueron ocasionados por las parejas de las victimas (párr. 6), los celos, el sentido de poder, pertenencia, falta de control de impulsos, consumo de sustancias estupefacientes, son algunos de los motivos relacionados al femicidio.

La naturalización de la violencia se origina en el contexto, es decir, existe una gran probabilidad de que los hombres que han vivido en ambientes violentos cometan en un futuro actos violentos, de la misma forma, mujeres que hayan vivido situaciones de violencia en su hogar pueden aceptar formas de violencia en la edad adulta.

\section{Consumo de alcohol y Violencia contra la mujer}

Las situaciones de ingesta de la sustancia causan daño a nivel personal, familiar y social, uno de los factores más observables se da en familias donde la pobreza y la falta de educación van de la mano, históricamente el consumo de bebidas alcohólicas ha sido vinculado a comportamientos violentos que ponen en riesgo la salud física y mental de las personas y en mayor medida el de las mujeres, esto debido a que la mayoría de los agresores conviven en un mismo lugar con las víctimas. En este sentido: Smarandache y Leiva (2019) afirman:

La violencia y el maltrato familiar se han reconocido como un problema socialmente vinculado al consumo de alcohol (...) Específicamente sobre la violencia contra la mujer los datos mundiales, revelan que ocurre en todos los países; esta es aplicada en granparte de la población femenina, tanto adulta como infantil (pp.45 y 46).

Un análisis más actual sobre esta problemática la realizo La Organización Panamericana de la salud (OPS) quien apoyó al Proyecto Genasis (Género, Alcohol y Cultura) para determinar la relación del consumo de alcohol y la violencia a la mujer, participaron en el estudio Argentina, Canadá, Costa Rica, Estados Unidos, México y Uruguay, Belice, Nicaragua, Perú y Brasil para medir la relación entre el consumo de alcohol y la agresión a la pareja de los resultados se concluye OPS y Proyecto Genasis (2010):

En cuanto a la relación entre el patrón de consumo de alcohol y la agresión en la pareja, los resultados mostraron que los bebedores tenían mayores probabilidades que los abstemios de notificar agresión en la pareja (como víctimas y como agresores) y que quienes bebían más por ocasión tenían mayores probabilidades de verse envueltos en agresión que quienes bebían menos (p. 52).

La violencia que genera el hombre hacia la mujer añadido al consumo de alcohol es por ahora considerado un problema de Salud Pública debido a que pone en evidencia el sufrimiento a la persona, la familia y la comunidad, por cuanto es importante su estudio, para generar mayores estrategias de intervención, con esto evitar más muertes y la reincidencia de los actos violentos. Villanueva (2013) afirma que principalmente la persona más afectada por la conducta violenta de los consumidores de alcohol ocasionales y habituales es la conviviente, (p. 47) por tanto mientras no exista rehabilitación para el agresor y la víctima, el circulo de la violencia continua. 
La evidencia también señala al consumo de alcohol como un factor de riesgo para el incremento de actos violentos, y manifiesta la diferencia señalando que la persona que es violenta, lo va a ser con consumo de alcohol o sin ingesta del mismo, caso contrario a las personas que únicamente infieren en conductas violentas cuando consumen bebidas alcohólicas, así, Mejía, Ochoa, Ríos, Yaulema y Veloz (2019) un estudio sobre los Factores de riesgo e indicadores de violencia de género en mujeres socias de bancos comunitarios en Chimborazo; concluye que un factor de riesgo individual que desencadena violencia es la presencia de consumo de alcohol, y que entre el $20 \%-21 \%$ de mujeres ha sido víctima de violencia psicológica y sexual por parte de su pareja (p. 7).

\section{Consumo del alcohol y violencia contra la mujer en la emergencia sanitaria de la COVID-19}

Durante la emergencia sanitaria en torno a la COVID-19 la población a nivel mundial fue confinada a sus hogares, a esta medida de prevención se suman otros factores que se van presentando paralelamente al tiempo de encierro, entre ellos temor al contagio, miedo a la muerte, noticias alarmantes, medidas gubernamentales que buscan sobrellevar la crisis afectando a la población, en este sentido se evidencia que el consumo de alcohol aumentó en pandemia; en Argentina, el periódico Infobae (2020) publica: "Según una encuesta realizada por el Consejo Nacional de Investigaciones Científicas y Técnicas (Conicet), el 45\% de las personas bebió más alcohol durante este tiempo que antes del aislamiento". (párr.4). Al analizar los resultados se verifica que el consumo responde a la existencia de mayor tiempo libre, desorganización en la planificación de actividades, factores emocionales y aburrimiento que afecta a la población, situaciones que no se manifestaban con agudeza antes del confinamiento debido a que la mayoría de la población contaba con recursos sociales (trabajo, estudios, etc); donde se sobrelleva lo antes mencionado.

Durante la pandemia Estados Unidos aplicó una encuesta a 1000 adultos, con el interés de investigar si la población durante la COVID-19 aumentó el consumo de sustancias, los resultados exponen que el $88 \%$ de la población consumió alcohol, el resto otras sustancias; entre las causas del consumo la población justifica la ingesta de sustancias por estrés, aburrimiento, ansiedad y depresión. (Marrero, 2020). De esta manera se confirma que el consumo de alcohol aumentó durante la pandemia en respuesta a factores estresantes como la pérdida de empleos, cierre de negocios, deudas, entre otros; que a su vez afectó de manera observable el cambio en la salud mental, síntomas ansiosos y depresivos que ocultaron las estrategias de afrontamiento y con ello el consumo de bebidas alcohólicas.

Ahora bien si la cuarentena fue estresante para la población general, más para las personas que son consumidores abusivos y dependientes, Ponciano (2020), afirma que: la cuarentena es estresante para todos, pero para las personas que consumen cualquier sustancia psicotrópica la forma de enfrentar el estrés es el consumo (p.6), sin embargo, el confinamiento trajo consigo la prohibición de ventas de bebidas alcohólicas, el cierre inmediato de bares, discotecas, cantinas, así como también suspensión de reuniones sociales; no obstante aumentó el consumo, esto quiere decir que la población buscó la forma de socializar, o liberar factores estresantes, quizá con su familia, amigos, vecinos, o simplemente consumió en soledad, visto de esta manera, el alcohol fue utilizado como fuente de alivio sintomático en respuesta a factores estresantes. 
En la otra línea, las denuncias contra la mujer aumentaron durante la pandemia, aunque también es cierto que muchas mujeres, víctimas de violencia, guardan silencio por diversas causas, una de ellas la violencia psicológica que el agresor ejerce sobre su víctima, al respecto Medina (2020) afirma que "En las entrevistas con las mujeres agredidas, los equipos de psicólogos detectaron que son víctimas de chantajes, amenazas y manipulación", en consecuencia se considera que son pocas las denuncias receptadas y se esperan más denuncias una vez finalizada la emergencia sanitaria, sin embargo, datos de algunos países de acuerdo a las llamadas de emergencias recibidas durante la COVID 19, refieren:

En Singapur y Chipre han registrado un incremento de más del $30 \%$ en las llamadas. En Nueva Gales del Sur (Australia), los trabajadores de primera línea comunicaron un $40 \%$ más de solicitudes de casos de violencia. En Francia, los casos de maltrato aumentaron un 30\% desde el inicio del confinamiento el 17 de marzo. En Argentina, las llamadas de auxilio por violencia doméstica han aumentado un $35 \%$ desde el 20 de marzo cuando se inició la cuarentena (...) En el Reino Unido, las llamadas, los correos electrónicos y las visitas a la página web de Respect, una organización benéfica nacional que lucha contra la violencia de género ha aumentado un $97 \%$, un $185 \%$ y un $581 \%$ respectivamente. En las tres primeras semanas de confinamiento, catorce mujeres y dos niños fueron asesinados en el país. (ONU, 2020)

Estas cifras son alarmantes y responden a las primeras semanas de confinamiento; el peligro que vive la mujer es elevado, vive amenazada en su propio entorno familiar, el permanecer todo el día en casa, las actividades domésticas, el cuidado de los hijos, la falta de ingresos económicos al hogar pueden ser motivos para que se produzcan más actos violentos a nivel familiar, Ante esto Fernández (2020) afirma "el confinamiento las ha puesto en peligro porque las familias han vuelto a la esfera privada, al aislamiento de la mujer en una situación amenazante tiene más dificultades para pedir ayuda" ( mayor al señalado.

La realidad no es muy diferente en Ecuador, país que confirma también que la violencia a la mujer aumentó durante la pandemia, los pedidos de auxilio han sido frecuentes al EC U 911 (línea que atiende y provee servicios emergentes a la población), según menciona el director del Servicio Integrado de Seguridad ECU, Ing. Juan Zapata (2020):

Desde el 12 de marzo, fecha en la que inició la emergencia sanitaria, hasta el 31 de mayo, se han atendido 28.367 episodios relacionados con temas de violencia intrafamiliar, lo que se traduce en 268 coordinaciones diarias realizadas por el ECU 911 tras pedidos de ayuda hechos a través del 9-1-1. (párr. 2)

Al respecto se menciona que, la mayor cantidad de reportes se recibieron durante los fines de semana y da a conocer que se receptaron 3.781 casos de violencia física, 17.911 casos de violencia psicológica y de connotación sexual 39 incidentes. Estos resultados corresponden a dos meses y medio de confinamiento, no se especifican las causas de la violencia, sin embargo, al igual que en otros países la mujer se ve lesionada en sus derechos, esto debido al desconocimiento de la ley, o al miedo ejercido por el violentador, o simplemente porque muchas han normalizado la violencia.

Por otro lado, los feminicidios también han estado presentes en Ecuador durante la pandemia sanitaria; al respecto: "Cada 72 horas una mujer, niña o adolescente es víctima de la violencia femicida en el Ecuador. En el $72 \%$ de los casos, los agresores 
eran parte del círculo familiar de las víctimas, exparejas o parejas" (El Comercio, 2020). La violencia a la mujer en Ecuador se ha venido haciendo evidente en el transcurso de los años, sin embargo, los reportes crecieron desde marzo 2020. En la misma línea:

La Asociación Latinoamericana para el Desarrollo Alternativo (Aldea) documenta 81 crímenes entre el 1 de enero y el 4 de octubre. Siete de cada 10 feminicidas eran parejas y exparejas. Cinco de cada 10 víctimas dejan hijos en la orfandad. El covid19 ha forzado a mujeres a convivir con sus agresores: 62 feminicidios desde el 16 de marzo, cuando se declaró la excepción, registran Aldea. Una tragedia. 69436 emergencia por violencia intrafamiliar recibió el ECU 911 entre el 12 de marzo cuando se declaró la emergencia sanitaria- y el 27 de octubre. (El Comercio, 2020)

De esta forma se visibiliza que la pandemia y el confinamiento es un factor de riesgo que agudiza situaciones de violencia, de cualquier tipo de violencia, la enfermedad llegó de manera tan imprevista que no se contaba con planes de atención emergentes que se pongan en marcha para continuar con el trabajo en torno las denuncias, las sentencias, el tratamiento a la víctima y la rehabilitación del agresor, por cuanto es importante que los gobernantes ahora trabajen en implementar protocolos de atención y políticas públicas con la finalidad de que se evite poner en riesgo la integridad física y mental de las personas.

\section{CONCLUSIONES}

A partir de la revisión bibliográfica realizada se puede concluir que el consumo de alcohol se ha naturalizado al igual que la violencia contra la mujer, esta realidad se torna más compleja ante la presencia de factores estresantes, poniendo en riesgo la integridad personal y social. Se ha visto la iniciativa de ciertas instituciones para enfrentar esta problemática, siendo una situación que genera malestar y que por tanto debe existir comprometimiento de todos quienes conformamos una determinada sociedad y cultura. Es así como continuando con el proceso de revisión bibliográfica se despliegan tres conclusiones en referencia a cada uno de los temas.

La información recopilada manifiesta que la mujer es vulnerada de manera imperante en sus derechos, así como también a pesar de la poca información sobre el consumo de bebidas alcohólicas, las fuentes analizadas datan que el consumo de alcohol ha aumentado tanto a nivel nacional y mundial, durante la emergencia sanitaria de la COVID-19, por tanto, se entiende que la violencia ejercida en contra de la mujer también ha aumentado en tiempo de pandemia.

El apartado del consumo de alcohol durante la emergencia sanitaria de la COVID19 informa que el consumo no disminuyó pese a que hubo restricción de movilidad y limitación del expendio de bebidas alcohólicas. La restricción en la venta de licor fue una decisión que tomaron los gobernantes en Ecuador, con la idea de que los actos públicos decrecieran y con eso subjetivamente también disminuya el consumo de alcohol, pero al analizar los datos de otros países se puede inferir que hubo ventas clandestinas, esta situación evidencia que se requiere mayor control en el expendio de bebidas, así como también se ve la necesidad de continuar con la sensibilización a la población. En la investigación se detectó que la población se afectó emocionalmente, presentando estrés, depresión, ansiedad, debido a la falta de empleo, falta de recursos económicos para la manutención del hogar, frustración, inclusive miedo al contagio y a la muerte; otra buena parte de la población pudo exacerbar los síntomas emocionales preexistentes a la emergencia sanitaria. En este 
sentido el consumo de alcohol fue para la población una fuente de alivio ante factores estresantes por lo tanto se minimizaron los riesgos de su ingesta.

La violencia en contra de la mujer durante el confinamiento evidencia que la mujer ha sido víctima de violencia, física, psicológica y sexual, trayendo consigo miedo, incertidumbre, síntomas depresivos-ansiosos, estrés, trastornos en la conducta alimentaria e inclusive ideación suicida. Los datos expresan que existió amento de violencia generada por la pandemia de la COVID-19, éstos fueron captados por las líneas de emergencia tanto a nivel mundial como nacional. El estado ecuatoriano debe asumir su responsabilidad en el cuidado a la integridad personal, vida y seguridad de la mujer. Es necesario el diseño e implementación de políticas de protección a la mujer, que se cree planes preventivos a mayor escala, se dé capacitación a los profesionales encargados de trabajar con esta problemática, y se contraten más profesionales para los servicios de salud y trabajo con la comunidad.

\section{REFERENCIAS}

ACNUDH, Alto Comisionado de las Naciones Unidas para los Derechos Humanos. (20 de diciembre de 1993). Declaración sobre la eliminación de la violencia contra la mujer. 85a sesión plenaria. Recuperado de https://www.ohchr.org/sp/professionalinterest/pages/violenceagainstwomen.aspx

Ahumada-Cortez, J. G., Gámez-Medina, M. E., y Valdez-Montero, C. (2017). El consumo de alcohol como problema de salud pública. Ra Ximhai, 13(2), 13-24

Castillo-Bustos, M. R. (2021). Técnicas e instrumentos para recoger datos del hecho social educativo. Retos de la Ciencia. 5(10), pp. 50-61.

Consejo de Protección de Derechos del Distrito Metropolitano de Quito (15 de abril 2020) El consumo del alcohol puede ser un detonante de mayor violencia intrafamiliar y de género: Es necesario limitar su consumo. Consultado en agosto del 2020. https://proteccionderechosquito.gob.ec/2020/04/15/8697605/

El Comercio. (29 de octubre 2020). Pandemia en la sombra. https://www.elcomercio.com/blogs/derechos-y-humanos/pandemia-sombra-femicidioaldea-pandemia.html

El Comercio. (9 de septiembre de 2019). Ecuador registra más de 60 femicidios en 2019 según plataforma de seguimiento. https://www.elcomercio.com/actualidad/ecuadorfemicidios-violencia-mujer-genero.html

Europapress. (16 de marzo de 2020). Los homicidios por violencia de género pueden aumentar tras el confinamiento por el coronavirus, según un experto [Mensaje en un blog]. https://www.europapress.es/epsocial/igualdad/noticia-homicidios-violenciagenero-pueden-aumentar-confinamiento-coronavirus-experto-20200316191441.html

Ferrat C.Y., Fernández E.H., González GMC, et al. (2019). Caracterización de las personas consumidoras de bebidas alcohólicas. Acta Med Cent. 13(4):523-531.

Fernández, R. I. (2020). Violencia de género en tiempos del Covid-19.

Graham, K., Bernards, S., Munné, M., y Wilsnack, S. (2010). El brindis infeliz: El consumo de alcohol y la agresión entre parejas en las Américas. Publicación científica 631. 1-258. ISBN: 978-92-75-31631-3

Lorente Miguel. (2020). Violencia de género en tiempos de pandemia y confinamiento. Revista Española de Medicina Legal, 46 (3) https://doi.org/10.1016/j.reml.2020.05.005

Llopis Giménez, C., Rodríguez García, M. I., y Hernández Mancha, I. (2014). Relación entre el consumo abusivo de alcohol y la violencia ejercida por el hombre contra su pareja en la unidad de valoración integral de violencia de género (UVIVG) de Sevilla. Cuadernos de Medicina Forense, 20(4), 151-169.

Marrero, E. (10 de septiembre de 2020). Aumenta el uso de alcohol y las drogas durante la pandemia de la COVID-19. Baptist Health South Florida. 
https://baptisthealth.net/baptist-health-news/es/aumenta-el-uso-del-alcohol-y-lasdrogas-durante-la-pandemia-de-la-covid-19/

Medina, F. (22 de septiembre 2020) La pandemia agravó la violencia psicológica. El comercio. https://www.elcomercio.com/actualidad/pandemia-agravo-violencia-psicologicaecuador.html

Mejía, M., Ochoa, D., Ríos, P., Yaulema, L., \& Veloz, S. (2019). Factores de riesgo e indicadores de violencia de género en mujeres socias de bancos comunitarios en Chimborazo Ecuador. Revista espacios, 40(32-2019).

Noroña, C. (25 de octubre 2020). Violencia de Género, la pandemia oculta: organizaciones sociales registran 81 femicidios en Ecuador desde enero hasta el 4 de octubre del 2020. El Comercio. https://www.elcomercio.com/actualidad/femicidios-violenciamujeres-mapa-

ecuador.html?fbclid=IwAR3RRW05Mi9F2MEZIJUrO8vWGWCEDjPAx5JChvWAQ7i8 L2ZTFxFCJN72G9k

OMS, Organización Mundial de la Salud. (21 de septiembre de 2020). El consumo nocivo de alcohol mata a más de 3 millones de personas al año, en su mayoría hombres. Ginebra, CH: OMS. https://www.who.int/es/news/item/21-09-2018-harmful-use-ofalcohol-kills-more-than-3-million-people-each-year--most-of-them-men

OMS, Organización Mundial de la Salud. (21 de septiembre de 2018). Alcohol: Datos y cifras. https://www.who.int/es/news-room/fact-sheets/detail/alcohol

ONU, Organización de las Naciones Unidas. (26 de junio de 2020). Víctimas de la violencia doméstica durante la padas pandemia. https://www.un.org/es/coronavirus/articles/un-supporting-trapped-domestic-violencevictims-during-covid-19-pandemic

ONU Mujeres, Organización de las Naciones Unidas Mujeres. (2012). Acabemos con la violencia contra las mujeres. https://www.unwomen.org/es/news/in-focus/endviolence-against-women/2012

OPS, Organización Panamericana de Salud. (5 de diciembre de 2008). Nuevo libro de la OPS revela vínculos entre consumo excesivo de alcohol y violencia doméstica. Washington, EU: $\quad$ Pan Health Organization. https://www.paho.org/hq/index.php?option=com_content\&view=article\&id=449:2008paho-book-establishes-new-links-between-excessive-alcohol-partnerviolence\&ltemid $=1926$ \&lang $=e s$

OPS, Organización Panamericana de Salud. (s.f.). Alcohol. Consultado en agosto del 2020. https://www.paho.org/es/temas/alcohol

OPS, Organización Panamericana de Salud y OMS, Organización Mundial de la Salud. (7 de abril de 2020). COVID- 19 y violencia contra la mujer. Lo que el sector y el sistema de salud pueden

hacer. https://iris.paho.org/handle/10665.2/52034

OPS, Organización Panamericana de Salud y OMS, Organización Mundial de la Salud. (2019). Informe sobre la situación mundial del alcohol y la salud 2018. Resumen. https://iris.paho.org/bitstream/handle/10665.2/51352/OPSNMH19012_spa.pdf?seque nce $=1$ \&isAllowed $=y$

OPS, Organización Panamericana de Salud y OMS, Organización Mundial de la Salud. (1992). Clasificación Estadística Internacional de Enfermedades y Problemas Relacionados con la Salud. CIE-10, 1(554).

Primicias. (12 de abril de 2020). 6.819 llamadas han recibido el ECU-911 por violencia de género. https://www.primicias.ec/noticias/sociedad/6819-llamadas-ecu911-violenciagenero/

Rosero, A. (20 de abril 2020) 186 delitos sexuales, denunciados en 20 días de aislamiento. El Comercio.https://www.elcomercio.com/actualidad/delitos-sexuales-denunciadosaislamiento-coronavirus.html. 
Servicio Integrado de Seguridad ECU 911. (s.f.) Gestión del Ecu 911 respecto a la violencia intrafamiliar. Quito, EC: Gobierno de la República del Ecuador. https://www.ecu911.gob.ec/gestion-del-ecu-911-respecto-a-violencia-intrafamiliar/

Vaiz Bonifaz, R. G., y Nakano, A. M. S. (2004). La violencia intrafamiliar, el uso de drogas en la pareja, desde la perspectiva de la mujer maltratada. Revista Latino-Americana de Enfermagem, 12(SPE), 433-438.

Villanueva, S. D. (2013). Violencia familiar asociado al consumo de sustancias psicoactivas en hombres que ejercen violencia. Avances en Psicología, 21(1), 35-46. 